Draft Version November 1, 2018

Preprint typeset using $\mathrm{L}^{A} \mathrm{~T}_{\mathrm{E}} \mathrm{X}$ style emulateapj v. 08/22/09

\title{
A 500 PARSEC HALO SURROUNDING THE GALACTIC GLOBULAR NGC 1851
}

\author{
Edward W. Olszewski, ${ }^{1,6}$ Abhijit Saha ${ }^{2,6}$, Patricia Knezek ${ }^{2,6}$, Annapurni Subramaniam ${ }^{3,6}$, \\ Thomas de Boer ${ }^{4,6}$, AND PATrick Seitzer ${ }^{5}$ \\ Draft version November 1, 2018
}

\begin{abstract}
Using imaging that shows four magnitudes of main sequence stars, we have discovered that the Galactic globular cluster NGC 1851 is surrounded by a halo that is visible from the tidal radius of $700 \operatorname{arcsec}(41 \mathrm{pc})$ to more than $4500 \operatorname{arcsec}(>250 \mathrm{pc})$. This halo is symmetric and falls in density as a power law of $r^{-1.24}$. It contains approximately $0.1 \%$ of the dynamical mass of NGC 1851 . There is no evidence for tidal tails. Current models of globular cluster evolution do not explain this feature, although simulations of tidal influences on dwarf spheroidal galaxies qualititively mimic these results. Given the state of published models it is not possible to decide between creation of this halo from isolated cluster evaporation, or from tidal or disk shocking, or from destruction of a dwarf galaxy in which this object may have once been embedded.

Subject headings: Galaxy:Globular Glusters:Individual:NGC 1851, Stars: Hertzsprung-Russell Diagram
\end{abstract}

\section{INTRODUCTION}

While it is clear that globular clusters must evaporate, and that globulars in a tidal field can also lose mass through their Lagrangian points, the exact state of disruption of many Galactic globulars is less certain. Much of the search for tidal structure (and for the fundamental parameters of globulars, for that matter) has been done with star counts from photographic plates (Grillmair et al. 1995, Leon et al. 2000, Illingworth \& Illingworth 1976 , Peterson and King 1975, and references therein). We give two examples of problems with the photographic datasets: NGC 288, for instance, has been claimed to have "extratidal structure" (Leon et al. 2000), while recent kinematic work finds no evidence for a coherent velocity gradient in the direction of the tidal tails (Kiss et al. 2007). Similarly, Da Costa \& Coleman (2008) use kinematics to put a limit of $0.7 \%$ of the mass of $\omega$ Cen between 1 and 2 tidal radii.

SDSS, HST, and mosaic cameras on large telescopes have allowed new insights into the structure of globular clusters. Recently several clusters have been shown to have spectacular tidal tails outside the tidal radius: Palomar 5 (Odenkirchen et al. 2001, Rockosi et al. 2002, Odenkirchen et al. 2003) and NGC 5466 (Belokurov et al. 2006, Grillmair \& Johnson 2006) are prototypes of this class of globulars. In addition, Lauchner et al. (2006) use SDSS to claim tidal tails in NGC 5053, though most of the stars are confined within the tidal radius. Sohn et al. (2003) use CFHT Mosaic imaging to discover "tentative detections of tidal halos" around Pal 3 and 4. The

\footnotetext{
Electronic address: eolszewski@as.arizona.edu

${ }^{1}$ Steward Observatory, The University of Arizona, Tucson, AZ

2 National Optical Astronomy Observatory, Tucson, AZ

${ }^{3}$ Indian Institute of Astrophysics, Bangalore, India

${ }^{4}$ Kapteyn Astronomical Institute, University of Groningen, The Netherlands

${ }^{5}$ Dept. of Astronomy, University of Michigan, Ann Arbor, MI

${ }^{6}$ Visiting astronomer, Cerro Tololo Inter-American Observatory, National Optical Astronomy Observatory, which are operated by the Association of Universities for Research in Astronomy, under contract with the National Science Foundation.
}

typical such cluster is "loose", with a relatively small ratio of tidal to core radius. Even those clusters that are convincingly being destroyed show the onset of two tidal tails at a relatively small distance from the tabulated cluster tidal radius.

It has been known for more than 40 years that the main bodies of globular clusters can be described by King (1966) models. Limiting (tidal) radii from these Kingmodel fits range from $1.6 \mathrm{pc}$ (NGC 6544, galactocentric distance $5.3 \mathrm{kpc}$ ) to $214 \mathrm{pc}$ (NGC2419, galactocentric distance $91.5 \mathrm{kpc}$ ), with a median of $36 \mathrm{pc}$ (derived from Harris 1996). 90\% of all Galactic globulars have radii of less than 100 pc (Harris 1996).

McLaughlin \& van der Marel (2005) have shown that Wilson (1975) models of the stellar distributions of globulars in the Milky Way, the Magellanic Clouds and the Fornax dwarf better describe the outer structures of thse clusters than do King models. McLaughlin and van der Marel (2005) homogeneously re-fit King, Wilson, and power-law models to 153 of these clusters. In general, the new King tidal radii are similar to those in the online Harris catalog, while the Wilson tidal radii are larger. Typically, the Wilson tidal radii are a factor of 2.5 larger than the King tidal radii (McLaughlin and van der Marel 2005), while for NGC 1851 the Wilson radius is a factor of 6.8 larger (McLaughlin and van der Marel's King tidal radius for NGC 1851 is 6.7 arcmin as opposed to 11.7 in the Harris compilation, with a Wilson tidal radius of 45 armin). We will use the King tidal radii in this paper simply because they are better known, and since in the case of NGC 1851 we believe that the fundamental data need improvement (star counts only extend to 10 arcmin from the center) to determine a realistic limiting radius. For the rest of this paper, we will use the phrase "tidal radius" to mean the King tidal radius tabulated in the online Harris catalog unless stated otherwise. Both the King tidal radius and the Wilson tidal radius are typically beyond where the star counts end, and can be poorly constrained in specific globulars.

What is the outer structure of a typical globular? Un- 
til recently, NGC 1851, the subject of this paper, would have been such a cluster: its absolute magnitude of -8.3 is 1 mag brighter than the median Galactic globular, and the tidal radius of $41 \mathrm{pc}$ is very close to the the median. NGC 1851, however, has recently been shown (Milone et al. 2008), using HST imaging, to be a most remarkable object. Above $\sim \mathrm{m}_{F 606 W}=19$, there are two distinct main-sequences (MS) and lower subgiant branches (SGB) visible, making it one of a small number of clusters with dual stellar populations. Milone et al. (2008) see no relative changes in the 2 subgiant populations out to a distance of 1.75 arcmin. Zoccali et al. (2009) study the radial extent of the two subgiant branches from groundbased data, and claim that the fainter sequence dies away 2.4 arcmin from the center (tidal radius is 11.7 arcmin), though Milone et al. (2009) refute the Zoccali work. Dinescu et al. (1997) derive the absolute proper motion for NGC 1851, which when coupled with the large (320.5 $\mathrm{km} / \mathrm{s}$ ) heliocentric radial velocity and current distances $\left(\mathrm{R}_{\odot}=12.1 \mathrm{kpc}, \mathrm{R}_{g c}=16.7 \mathrm{kpc}, \mathrm{X}=-4.3 \mathrm{kpc}, \mathrm{Y}=-8.9\right.$ $\mathrm{kpc}, \mathrm{Z}=-6.9 \mathrm{kpc}$ ), gives an orbit with period $\sim 0.4 \mathrm{Gyr}$, apogalacticon distance $\sim 30 \mathrm{kpc}$, and perigalacticon distance $\sim 5 \mathrm{kpc}$. This orbit passes through the disk of the Milky Way five times per Gyr at distances ranging from 5 to $30 \mathrm{kpc}$ (orbital calculations graciously performed by Piatek (2009)). According to the theoretical papers cited in the discussion, disk shocking and tidal shocking should be important for NGC 1851, and a tidal tail should be seen. Leon et al (2000, their figure 10) claim to see symmetric structure to about 20 arcmin radius (about $1.7 \times$ the tidal radius) and then "extensions which are likely tracing the orbital path..." Leon et al (2000) azimuthally average background-count subtracted data and fit a power law to radially distant points, finding a power law slope for NGC 1851 of -0.98 , similar to what we find in Section 2. Our new observations contradict these claims: we see no tidal tails and do not see discrete structures in common with the photographic counts, but show smooth extended structure.

\section{OBSERVATIONS AND DATA REDUCTION}

We discovered that NGC 1851 has extended structure while observing a control field, centered 3.5 tidal radii from NGC 1851, for a project to probe the outer structure of the LMC. The $\mathrm{R}$ versus $\mathrm{C}-\mathrm{R}(\mathrm{C}$ is Washington $\mathrm{C}$ (Canterna 1976), centered at $\sim 4000 \AA$ ) color-magnitude diagram (CMD) of this field $\mathrm{C} 18(\mathrm{l}=245, \mathrm{~b}=-35)$ is compared to $\mathrm{C} 20(\mathrm{l}=245, \mathrm{~b}=-25), 10$ degrees away, in Figure 1. A main sequence is obvious in $\mathrm{C} 18$ from $\mathrm{R} \sim 18.5$ to $\mathrm{R} \sim 22$. C20, at lower latitude, has more stars overall, but does not show this additional feature. This feature is most likely a main sequence of an object at a single distance, and corresponds exactly to the main-sequence locus in published NGC 1851 main body photometry (e.g., Figure 10 in Walker 1992), after a reasonable color transformation.

We therefore began to map out this main sequence by acquiring nine fields outlining the cluster from $\sim 1.0-\sim 6.5$ tidal radii. Exposures were 300 seconds in $\mathrm{V}$ and 600 seconds in B, using the CTIO $4 \mathrm{~m}$ Blanco telescope with the Mosaic II CCD array. Each field is $36 \times 36$ arcmin on a side. Table 1 gives a diary of observations of the BV fields.
Data were reduced using IRAF's ${ }^{7}$ MSCRED package and a set of homegrown IDL routines (Saha et al. (2010). Photometry is then done with a privately modified (by Saha) version of DoPHOT (Schechter et al. 1993) and with a set of post-DoPHOT programs written by one of us (Saha). Two aspects of the reduction are relatively uncommon: first, we create single resampled images from the 8-CCD mosaics as input to DoPHOT. Our extensive testing shows that the photometry is unaffected by our resampling of the images (Saha et al. 2010). Second, DoPHOT was run with a non-variable PSF. However, the real PSF does vary with position on the field-of-view, so the PSF fitted magnitudes have consequent position dependent trends of several percent. These were corrected by obtaining aperture magnitudes for the brighter high $\mathrm{S} / \mathrm{N}$ stars (which do not vary with position), and comparing the PSF fitted magnitudes to the aperture measured values, and thus deducing the position dependence of the fitted magnitudes. The derived corrections as a function of position on the field-of-view were then applied for all stars. This procedure is more robust for these data than using a variable PSF. These extra steps also allow for corrections due to tilt and piston and defocus as a function of position on each original CCD. There is demonstrably little chip-to-chip photometry shift remaining after using this technique. The small chip-to-chip and field-to-field photometry errors from these reduction procedures keep the photometry from wandering in zeropoint and scale, and permit the large-scale mapping described here.

The nine fields are combined by using stars in common between frames to adjust the instrumental magnitudes to a common scale, the scale of our innermost field. The photometric zeropoint of the innermost field comes from comparison of published NGC 1851 photometry (Walker 1992). In other words, photometry within $\sim 1$ tidal radius was forced to that of Walker (1992), but the main sequence outside that radius is bootstrapped to the magnitudes so obtained in the innermost field (field 2 shifted by using overlap region of fields 1 and 2, field 3 shifted by using overlap of fields 1 and 2 against it, etc.) Thus any excursions of main sequence structure over the extended field are preserved, if they exist. The upper plot of Figure 2 shows the CMD of the entire area imaged, including the small region inside 1 tidal radius. We see a clear main sequence and lower subgiant branch, the wall of thick disk and halo turnoff stars, the wall of $\mathrm{K}$ and $\mathrm{M}$ stars, and the blob of faint galaxies. The lower plot shows only stars more than 2000 arcsec from the cluster center (more than 2.85 tidal radii). In both CMDs there are two turnoffs and lower subgiant branches (Figure 3), extending the Milone et al. (2008) HST result to extraordinarily large radii. This result contradicts the Zoccali et al. (2009) claim that the fainter SBG disappears 2.4 arcmin from the cluster center, and is consistent with the recent Milone et al. (2009) result which follows the two subgiant branches out to at least 8 arcmin.

We define a region on the CMD that contains the main sequence and lower subgiant branch. This region is a band that follows the ridge line of the MS and SGB and

7 IRAF is distributed by the National Optical Astronomy Observatory, which is operated by the Association of Universities for Research in Astronomy (AURA) under cooperative agreement with the National Science Foundation. 

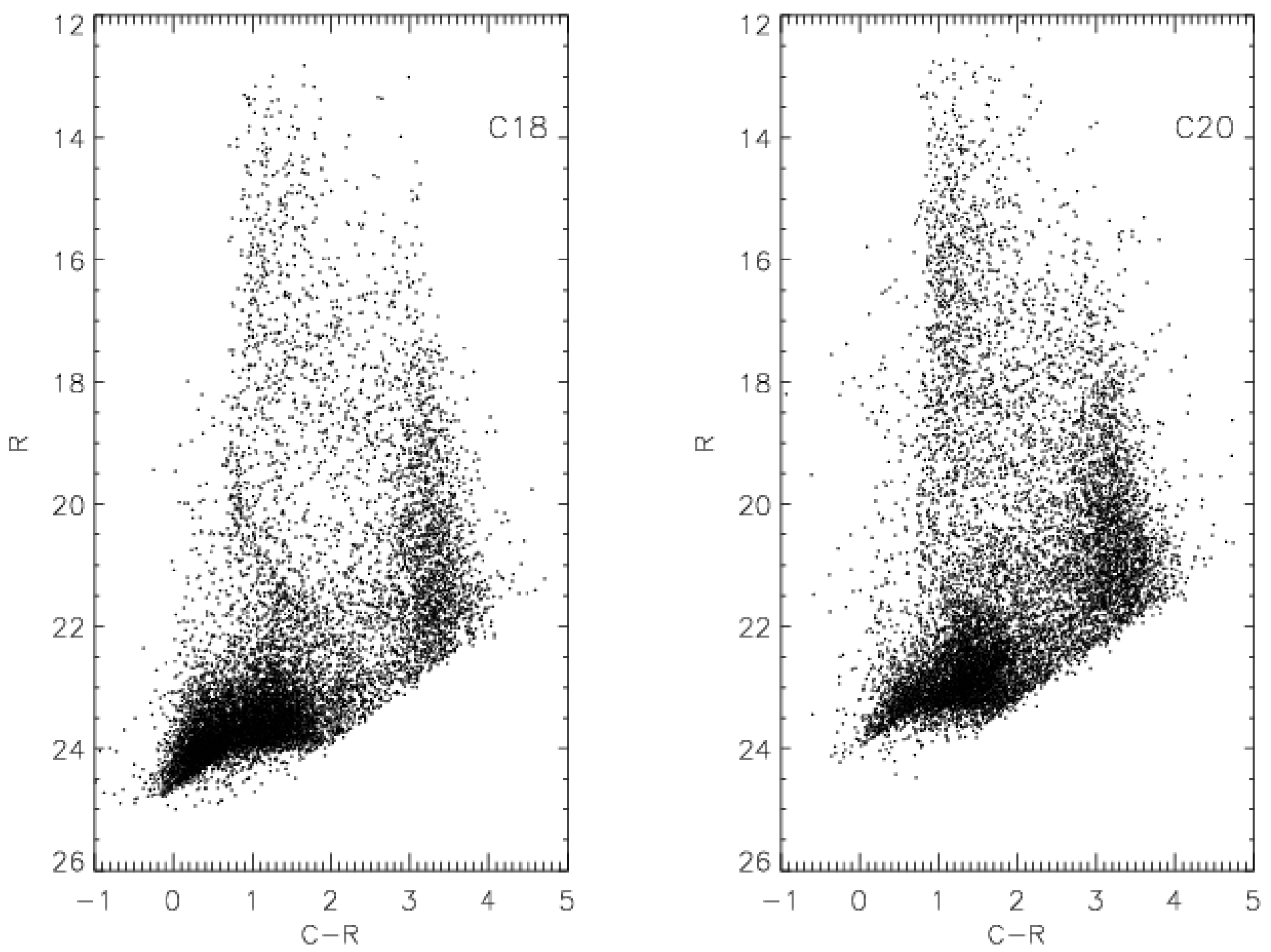

FIG. 1.- R versus C-R cmds for control fields C20 and C18 (near NGC 1851) from another project. Note the wall of F stars and the "extra" main sequence in C18.

TABLE 1

LOG OF BV OBSERVATIONS OF NGC 1851

\begin{tabular}{lccr}
\hline \hline \multicolumn{1}{c}{ Field } & RA J2000.0 & Dec J2000.0 & M.J.D. of V data \\
\hline NGC 1851 inner & $05: 15: 06.8$ & $-40: 26: 47$ & 54825.20172858 \\
NGC 1851 E & $05: 16: 57.1$ & $-40: 02: 51$ & 54715.40055975 \\
NGC 1851 W & $05: 11: 16.0$ & $-40: 03: 31$ & 54823.28117295 \\
NGC 1851 N & $05: 14: 07.1$ & $-39: 29: 57$ & 54822.33423601 \\
NGC 1851 S & $05: 14: 07.2$ & $-40: 35: 50$ & 54715.38609743 \\
NGC 1851 NE & $05: 16: 59.9$ & $-39: 29: 52$ & 54823.32000866 \\
NGC 1851 NW & $05: 11: 15.0$ & $-39: 29: 54$ & 54823.33312843 \\
NGC 1851 SE & $05: 16: 59.3$ & $-40: 35: 47$ & 54823.29429358 \\
NGC 1851 SW & $05: 11: 15.0$ & $-40: 35: 51$ & 54823.30709821 \\
& & & \\
\hline
\end{tabular}



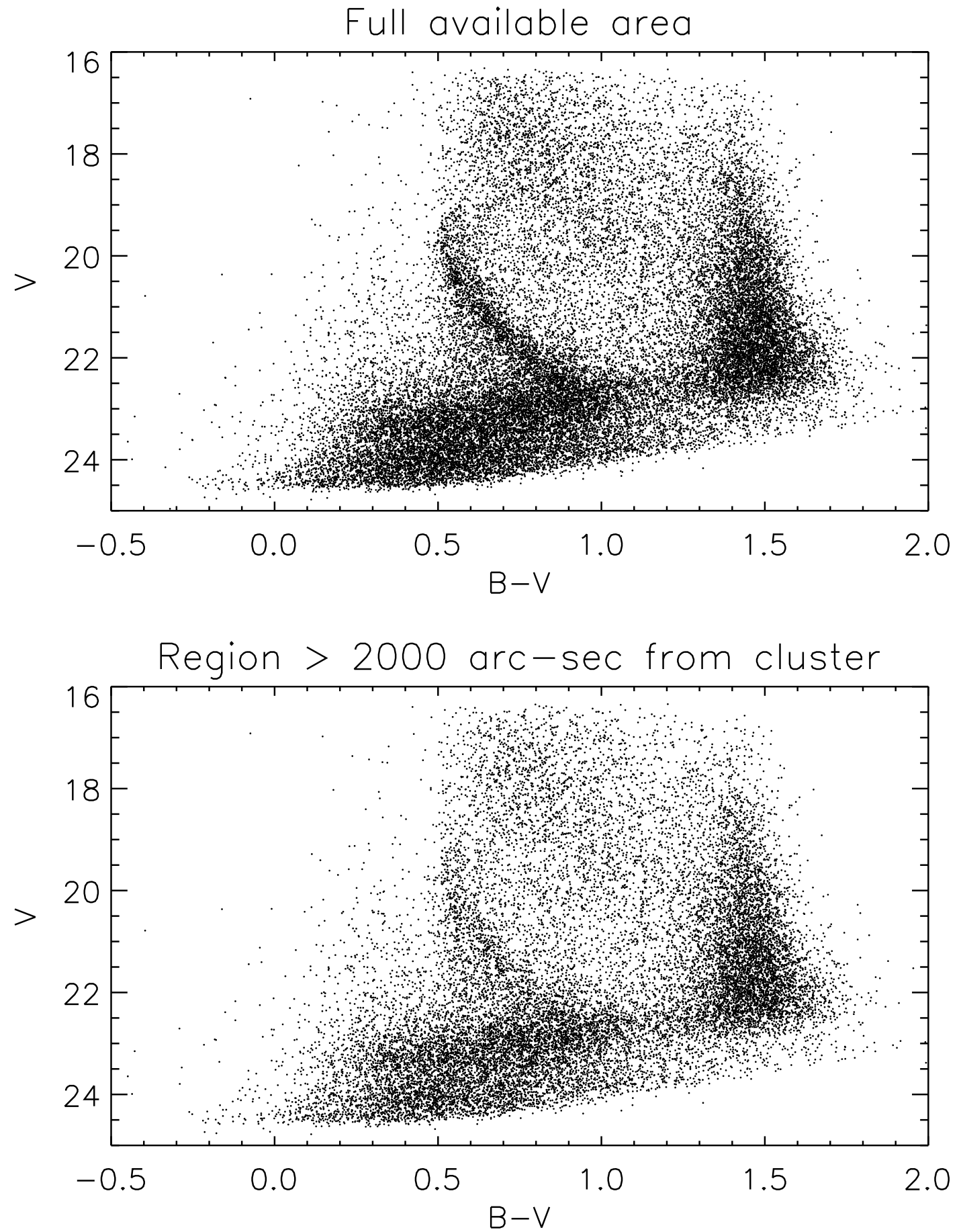

FIG. 2.- V versus B-V cmds for two regions surrounding NGC 1851. Top diagram is of all acquired data. Bottom figure is of a region more than 2.85 tidal radii from NGC 1851. In both cases the main sequence is identical to that of the globular, and the double subgiant branches are visible. 

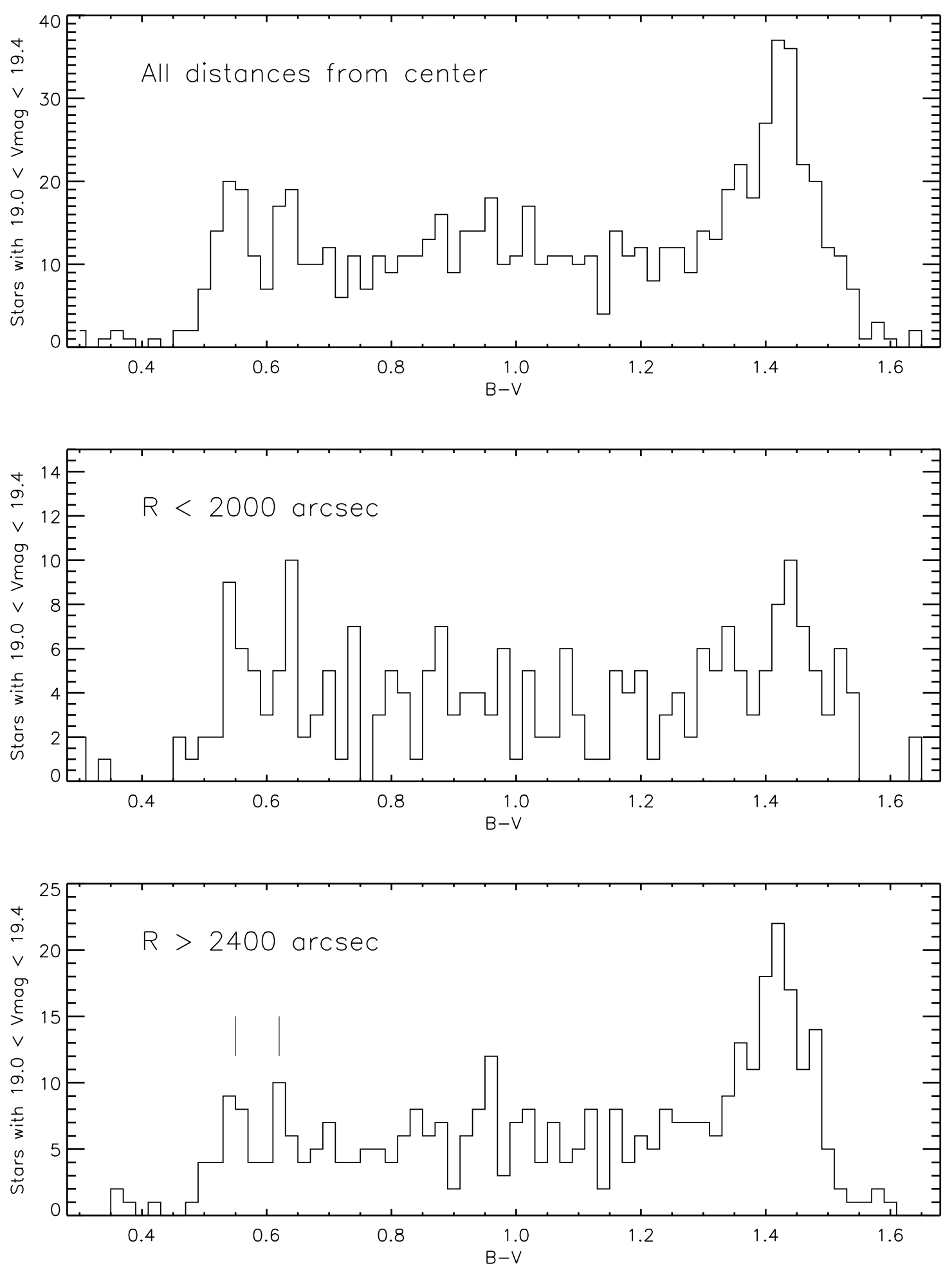

FIG. 3. - Histograms of all stars with $19.0<\mathrm{V}<19.4$, in $\mathrm{B}-\mathrm{V}$ bins of 0.02 , to show peaks in the CMD. The features at $\mathrm{B}-\mathrm{V}=0.55$ and 0.63 are the two stellar populations in NGC 1851. The peak at about $\mathrm{B}-\mathrm{V}=1.4$ is made up of the foreground $\mathrm{K}$ and $\mathrm{M}$ stars. Top: Histogram of all observed stars; Middle: Histogram of all stars closer than 2000 arcsec (117 pc); Bottom: Histogram of all stars more than $2400 \operatorname{arcsec}(140 \mathrm{pc})$ from the center to about $4000 \operatorname{arcsec}(230 \mathrm{pc})$. The two peaks of interest are marked with tickmarks in the bottom plot. 
is 0.15 mag wide in (B-V) (see Figure 4). Background estimates come from regions centered 0.15 mag redward and blueward of this box, and by scaling the C20 control field in Figure 1. In Figure [5 we plot the positions of stars in our MS and SGB regions readily distinguished in Figure 2. We also plot the distribution of stars in the two background regions of the CMD. Aside from a density peak of non-cluster stars at large distances to the East, there are no obvious trends. Figure 6 shows the angular distribution of stars outside the tidal radius, in $15^{\circ}$ bins, of stars in the main-sequence region of the CMD (dashed), in the two background regions of the CMD (dotted), and in the total number of NGC stars (solid), which is the counts in the dashed histogram minus half of the dotted. Counting errors for the solid curve are \pm 7 on average, which encompasses most of the bins. There is no evidence, therefore, for tidal tails or angular structure on the distribution of NGC 1851 stars.

Counting stars on the main sequence, and counting stars to the red and blue of the main sequence to use as background estimations, gives the density plot of Figure 7 We see steeply declining counts from about 500 arcsec to about 1000 arcsec $(1.3 \times$ the cataloged tidal radius), with a very shallow decline out to the limits of our imaging, more than 4000 arcsec. Fitting a straight line to this halo, beyond 1200 arcsec, gives $\log ($ counts $) \propto$ $\log (r)^{-1.24}$, with $1-\sigma$ errors on the power law of -0.58 and -1.9 . Figure 8 shows the density plot in linear units to underscore the large extent of the NGC 185 halo. While our discovery of stars out to 67 arcmin may not seem extreme if one believes the Wilson tidal radius of 45 arcmin for NGC 1851, we note that the King model fit using the code of McLaughlin and van der Marel (2005) gives no stars beyond 5.8 arcmin, and the Wilson model gives no stars beyond 43 arcmin. We can still see the main sequence by eye (Figure 21) at radii extending beyond the (very uncertain)Wilson tidal radius.

\section{DISCUSSION}

Unlike the case of the Pal 5 tidal tails, this set of extratidal stars does not make up a large fraction of the current mass of the cluster. For a region exterior to 13 arcmin from the center, we count 1647 total stars in the main sequence region of Figure 4. Using off-mainsequence regions defined in Figure 4 and in a scaled version of control field C20, we measure that 699 of those stars are field stars. The net number of stars outside 13 arcmin is therefore $948 \pm 40.5$, or $58 \%$ of the total. Despite the extremely low surface brightness of $\sim 32 \mathrm{mag}$ $\operatorname{arcsec}^{-2}$, the main sequence and the SGB remain vivid.

If the typical mass on this main sequence is $0.5 \mathrm{M}_{\odot}$, this halo of $500 \mathrm{M}_{\odot}$ measures $0.1 \%$ of the dynamical mass of NGC 1851. NGC 1851 is therefore embedded in a very low-mass halo 500pc across, or "dwarf spheroidal size". This halo also contains two populations, as seen in the two main sequences, of unknown origin.

The state of modelling of stars outside the King tidal radius and not in tidal tails, is unsatisfying. Observationally, as pointed out in Odenkirchen et al. (2009), the expected signatures of tidal mass loss are not as clear as hoped. Gnedin and Ostriker (1997) model the destruction rates of globulars, but do not provide detailed spatial stellar distributions. A number of papers (e.g., Spitzer 1940; Gnedin \& Ostriker 1997) show that mass loss, because of internal 2-body interactions that force lower-mass stars slowly to the periphery of the cluster, changes the mass-function from center to edge of the cluster. Combes et al. (1999) find that unbound stars (not in the tidal tails) change slope at the tidal radius, to a surface density slope of -3 . Combes et al. show a time sequence of their model $\mathrm{m} 2$ (their table 1 and figure 14). $\mathrm{m} 2$ has a tidal radius of $60 \mathrm{pc}$. Even after significant mass loss the inner symmetrical part stays at about 60 pc radius, with the tidal tails emanating from the tidal radius area. Fukushige \& Heggie (2000) use simulations to show that stars inside the tidal radius with energies above the escape energy can leave the cluster on a similar timescale to stars leaving due to 2-body interactions, approximately a Hubble time. This simulation might come closest to mimicking a cluster like NGC 1851, except for the complications due to the orbital eccentricity of NGC 1851. Mass loss in a slightly different context, that of dwarf spheroidal galaxies, has been studied by Johnston et al. (1999) and by Peñarrubia et al. (2008). Johnston et al (1999), in a study of the mass loss rates from dwarf spheroidal galaxies, point out the existance of a change of slope of the stellar density (star counts) at the radius where now-unbound stars become important. They derive a much shallower slope for the region outside the tidal radius, -1 or so, in contrast to that of Combes et al.

Typical observational cases might be those of NGC 2419 and M92. Ripepi et al. (2007), see main sequence stars in NGC 2419 out to more than 1.2 tidal radii. Lee et al. (2003) show the radial profile for M92, with power law fits of slope $\sim-1$ beyond the tidal radius, but with symmetric structure out to perhaps $1.5 \times$ the tidal radius, little evidence for tidal tails, and possible clumpy structure outside the symmetric structure. This is qualitatively consistent with Testa et al. (2000) whose analysis of POSS data shows symmetric structure out to perhaps $1.5-2 \times$ the tidal radius, plus more distant irregular structure. Testa et al. counted stars to 16 th magnitude in photographic F, while Lee et al. counted stars to $\mathrm{V}=20.0$ and to 23.5. Some of the radially distant structures are in common to both studies, at least qualitatively. However, Drukier et al. (2007) show that beyond the M92 tidal radius, almost no stars have the M92 velocity (their Figure $5)$.

In addition, good evidence for extratidal material in some globulars has been overlooked. Lee et al. (2004, their Figure 5) show the CMD of a control field at more than 2.5 tidal radii from NGC 7492 that clearly shows the cluster main sequence.

Given the state of the modelling described above, it is impossible to distinguish between creation of such a halo from 2-body internal interactions, disk shock heating, tidal heating, destruction of a primordial dwarf galaxy in which NGC 1851 was embedded, and initial conditions. Nonetheless, we briefly examine some of these.

Mass loss caused by Galactic tides is the obvious explanation for the long tidal tails of objects such as Pal 5 (Odenkirchen et al. 2001; Rockosi et al. 2002; Odenkirchen et al. 2003; Koch et al. 2004). The Pal 5 tidal tails emerge from the cluster at about the tidal radius of 16 arcmin, or $\sim 100 \mathrm{pc}$ (Odenkirchen et al. 2001, their Figure 2). We see no sign of tidal tails in the NGC 1851 halo out to the limit of our survey, a radius of 250 


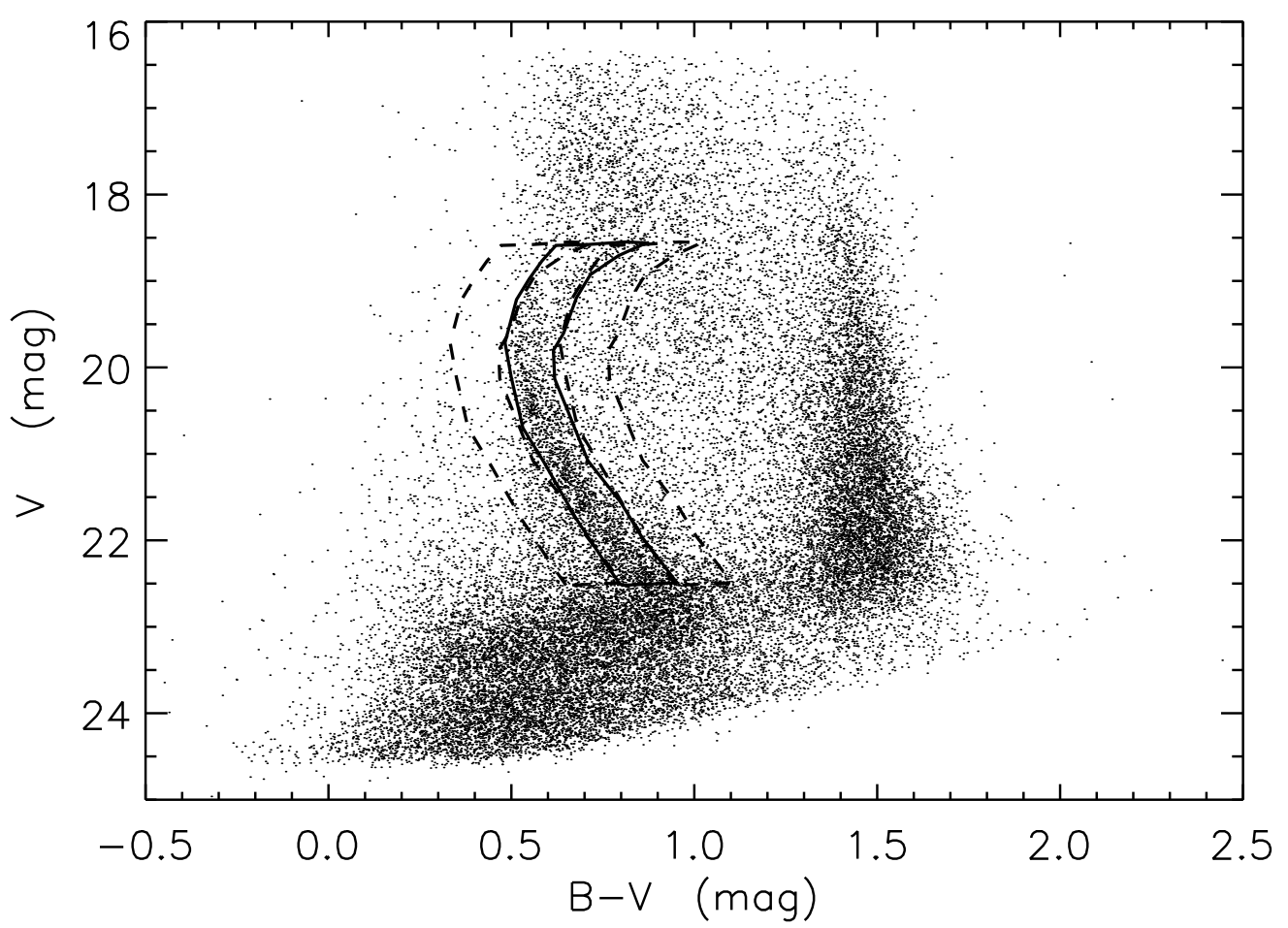

FIG. 4.- CMD of NGC 1851 halo with our main-sequence and subgiant regions, and the two off-sequence background regions overlaid. Each region is 0.15 mag wide in $\mathrm{B}-\mathrm{V}$. There is a very small overlap at $\mathrm{V}=18.5$.
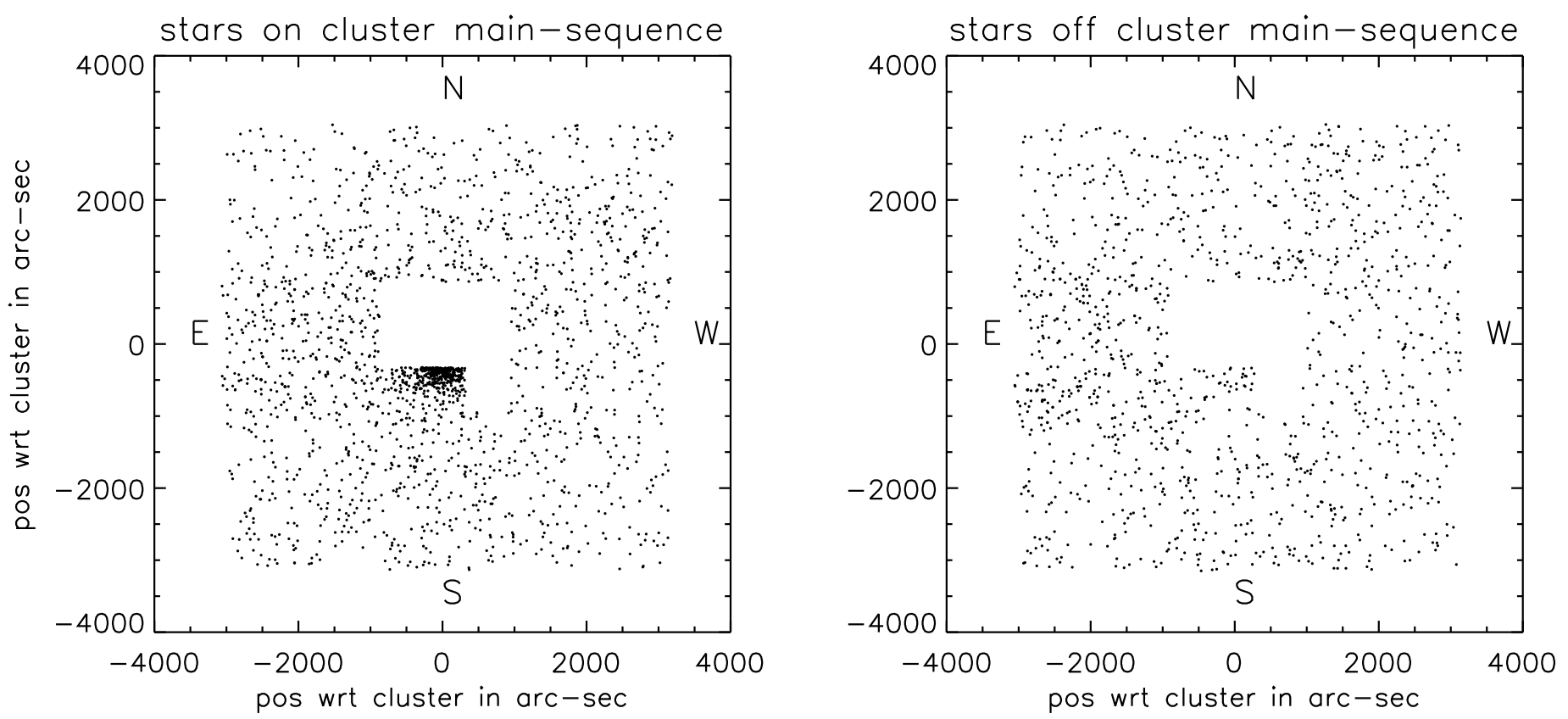

FIG. 5.- Left: positions of all stars that meet our criteria for being on the observed main sequence and subgiant branches. Regions interior to 1 tidal radius $(700$ arcsec) were not observed except for one small region to the south. The extreme plotted points are more than 6 tidal radii from the center. Right: the same plot for the distribution of stars in the two background regions. An excess of field stars can be seen to the East. 


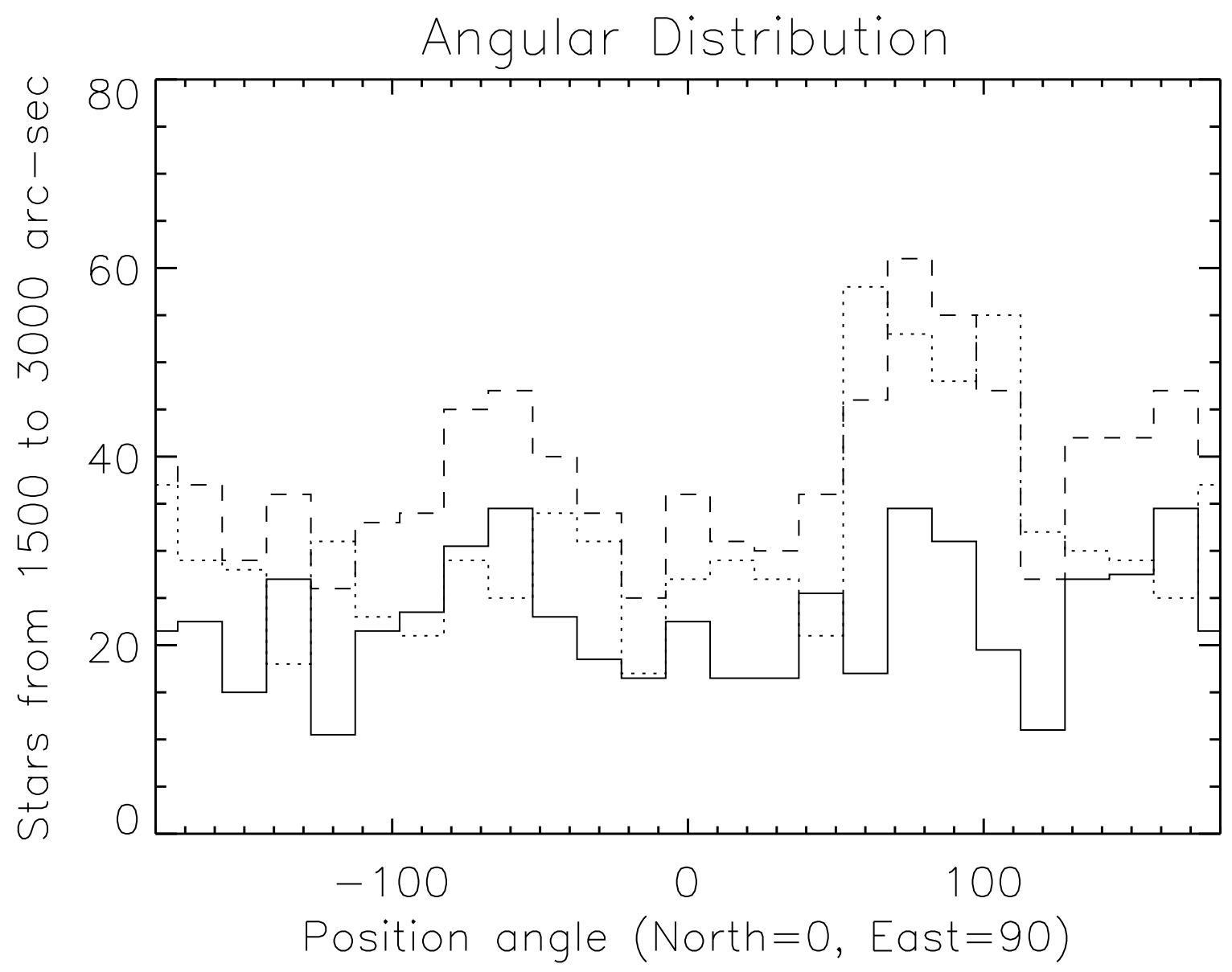

FIG. 6. - The angular distribution of stars as a proof of the lack of tidal tails. Dashed histogram: all stars outside of $\sim 1$ tidal radius, in the main sequence region of Figure 4 Dotted histogram: all stars in the two off-cluster positions (twice the area in the CMD) shown in Figure 4 Solid histogram: the difference "dashed" minus $0.5 \times$ " dotted" (there are two off-main-sequence regions). This is the total number of NGC 1851 stars in the main sequence region. The average is about $25 \pm 7$ for the solid histogram, showing the lack of statistically significant features in the azimuthal distribution. 


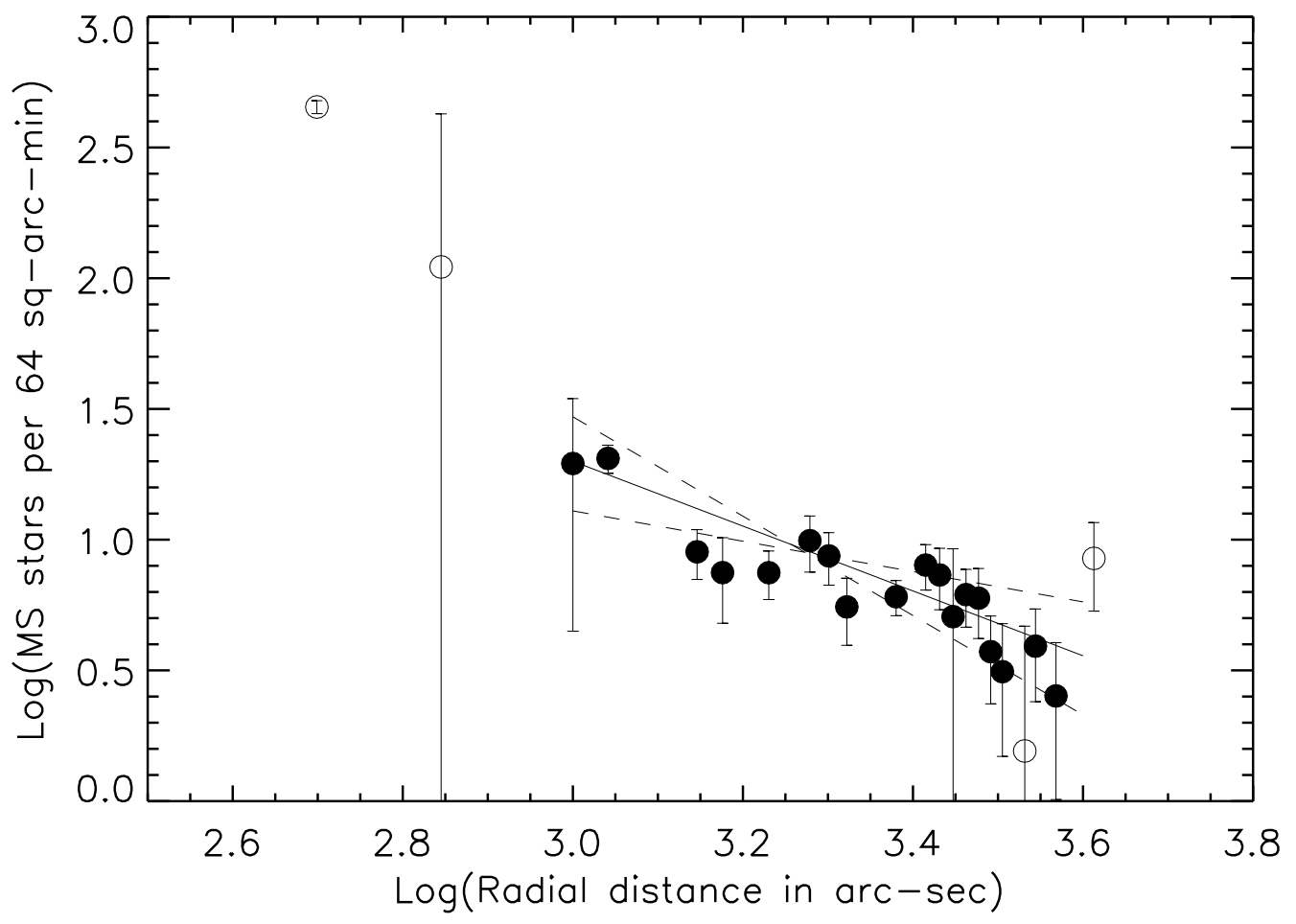

FIG. 7.- Density plot of stars surrounding NGC 1851. Note the Y scale of log of stars per $64 \operatorname{arcmin}^{2}$.Open circles are regions in radius with very incomplete coverage. Filled circles between 1200 and 4000 arcsec were used for power-law fits. Solid line is the best fit $\left(\chi^{2}=0.9\right)$ of $\mathrm{r}^{-1.24}$; dashed lines are the $1-\sigma$ limits of $\mathrm{r}^{-1.9}$ and $\mathrm{r}^{-0.58}$.

pc. The orbit of Pal 5 (Scholz et al. 1998), with $R_{\min }$ between 5 and $9 \mathrm{kpc}, R_{\max }$ between 17 and $18 \mathrm{kpc}$, and orbital period $0.4 \mathrm{Gyr}$, is qualitatively similar to that derived for NGC 1851 above. Since Pal 5 is much fluffier than NGC 1851, we would indeed expect that Pal 5 would be closer to dissolution than NGC 1851, as it is. Leon et al. (2000) claim evidence for tidal tails tracing NGC 1851's orbit, evident at distances of 15-40 arcmin (50-140 pc). Our CMDs contradict these claims.

Gnedin and Ostriker (1997) show that the evaporation time for NGC 1851 is very long, about 10 Hubble times. Gravitational shocking is also of order the same value. If the halo of NGC 1851 is simply an evaporated or shocked halo, there is qualitative consistency with $\mathrm{Pal}$ 5: Pal 5 (from their table 3) is quickly destroyed, while NGC 1851 is very slowly destroyed. The models do not provide the information necessary to compare our empirical halo with a model halo.

The studies of destruction of dwarf spheroidals (Johnston et al. 1999; Peñarrubia et al. 2008, their Figure 2) offer a possible explanation for the structure of NGC 1851. The Peñarrubia et al. paper models a dwarf spheroidal as an NFW dark-matter profile with a much more concentrated baryonic component. NGC 1851 would be an even-more concentrated nucleus in such a model. Peñarrubia et al. (2008, their figures 2 and 3) show the effect of stripping away most of the mass of the dSph. What remains is an object that can be fit to a King model, but with a shallow halo outside the place where the crossing time equals half the orbital time. One probably cannot extend these models to the remnants of a nucleated dwarf, so we must appeal to n-body experts to make the proper models for NGC 1851 . The most striking feature of NGC 1851 when compared to $\omega$ Cen, M54, and NGC 2419, clusters that have at one time been hoped to be remnant nuclei of nucleated dwarfs, is the small core radius, $0.2 \mathrm{pc}$, of NGC 1851. NGC 1851 (Yong et al. 2009, Ventura et al. 2009 ), similarly to $\omega$ Cen, does have a large spread in the elemental abundances $\mathrm{C}+\mathrm{N}+\mathrm{O}$, making it different from most globulars.

Of the 24 Galactic globulars more luminous than NGC 1851, the four clusters with the largest current galactocentric distances all have larger Wilson tidal radii than NGC 1851, and three have interesting associations with stellar streams: NGC 1851 is on an elliptical orbit reaching in to a galactocentric distance of $5 \mathrm{kpc}$ and out to 30 kpc (Section 1). NGC 2419 might be associated with the Virgo Stellar Stream, which is on an extremely elliptical orbit (Casetti-Dinescu et al. 2009). NGC 5824 may be associated with a stellar stream (Newberg et al. 2009). NGC 6715 is M54, the core of the Sgr dSph. The other three luminous clusters with larger Wilson tidal radii than NGC 1851 are interior to the current position of NGC 1851, with NGC 6139 the most extreme $\left(\mathrm{R}_{G C}=1.7 \mathrm{kpc}\right.$ and $\mathrm{R}_{\text {tidal }(\text { Wilson })}=676 \mathrm{pc}$. $)$ The orbit of 47 Tuc is rather circular in the XY plane $\left(\mathrm{R}_{a p o}=7.3\right.$ $\mathrm{kpc}, \mathrm{R}_{\text {peri }}=5.2 \mathrm{kpc}$ ), while M3's orbit has an ellipticity of $0.42\left(\mathrm{R}_{\text {apo }}=13.4 \mathrm{kpc}, \mathrm{R}_{\text {peri }}=5.5 \mathrm{kpc}\right)($ Dinescu et al. 1999). A parameter that relates luminous globulars to large physical size might therefore be some combination of short-period orbit, eccentric orbit, or association with a stellar stream. 


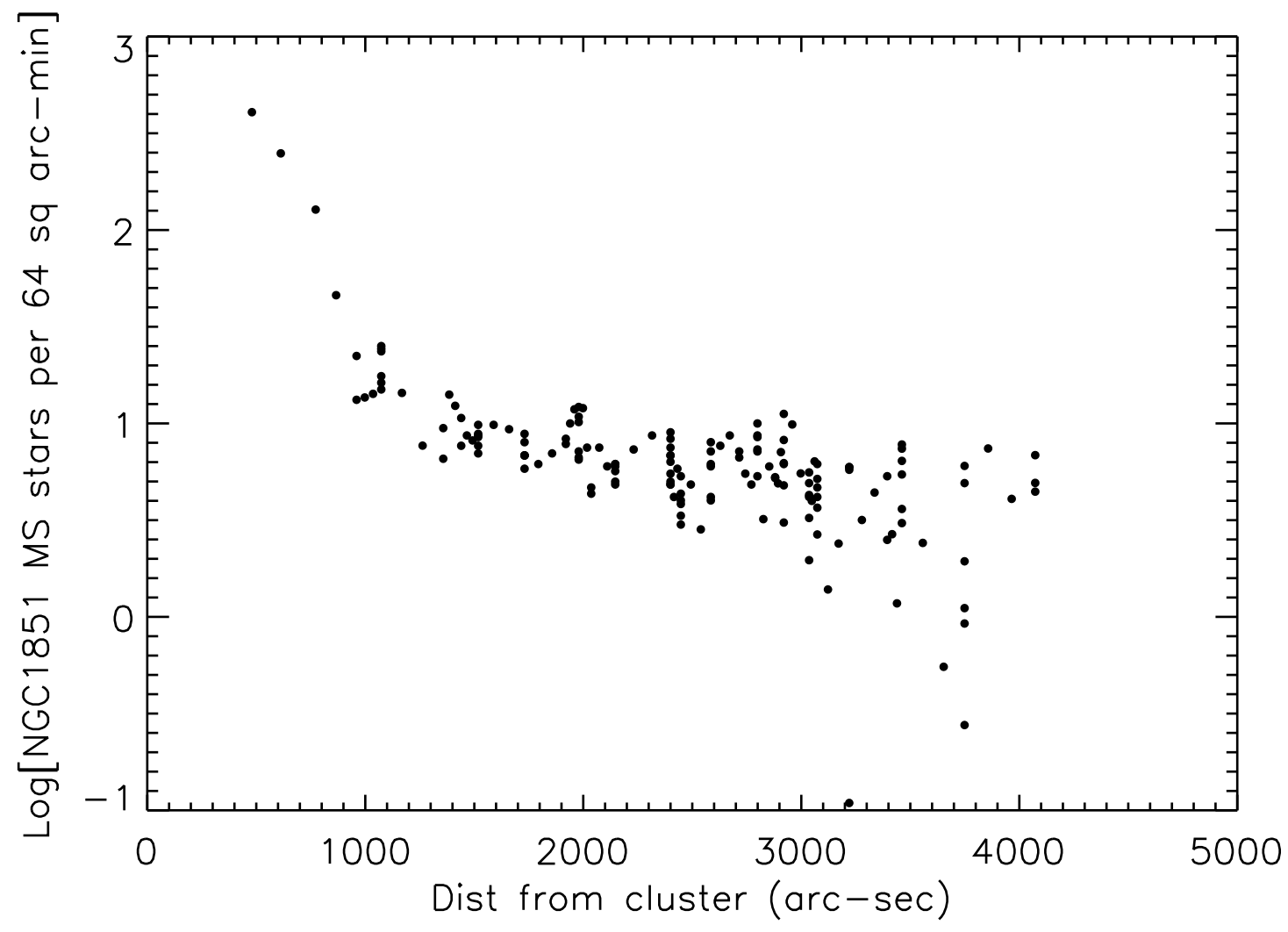

FIG. 8.- Density plot of stars surrounding NGC 1851. The $\mathrm{x}$ axis is now linear in radius.

While the correct explanation for the halo of NGC 1851 is presently elusive, the observational imaging result is clear and strong. Modern imaging and reduction techniques can therefore tell if NGC 1851 is an exceptional cluster and if the fundamental data for the 150 Milky Way globular clusters need to be much improved and extended with wide-field CCD photometry.

We thank the CTIO mountain staff for their help in observing, both on the mountain and from La Serena.
We also thank Chris Smith for computer, archive, wiki, and "MOSOCS" help. Slawomir Piatek performed the new orbital calculations for NGC 1851. Bill Harris introduced us to the McLaughlin and van der Marel paper, and Dean McLaughlin provided us with his fitting code and thoughtful comments on the quality of tidal radius determinations. We thank the referee for helpful comments. EO was partially supported by NSF grants AST-0505711 and 0807498.

\section{REFERENCES}

Belokurov, V., Evans, N. W., Irwin, M. J., Hewett, P. C., \& Wilkinson, M. I. 2006, ApJ, 637, L29

Canterna, R. 1976, AJ, 81, 228

Dinescu, D. I., Girard, T. M., \& van Altena, W. F. 1999, AJ, 117, 1792

Casetti-Dinescu, D. I., Girard, T. M., Majewski, S. R., Vivas, A. K., Wilhelm, R., Carlin, J. L., Beers, T. C., \& van Altena, W. F. 2009, ApJ, 701, L29

Combes, F., Leon, S., \& Meylan, G. 1999, A\&A, 352, 149

Da Costa, G. S., \& Coleman, M. G. 2008, AJ, 136, 506

Dinescu, D. I., Girard, T. M., van Altena, W. F., Mendez, R. A., \& Lopez, C. E. 1997, AJ, 114, 1014

Drukier, G. A., Cohn, H. N., Lugger, P. M., Slavin, S. D., Berrington, R. C., \& Murphy, B. W. 2007, AJ, 133, 1041

Gnedin, O. Y., \& Ostriker, J. P. 1997, ApJ, 474, 223

Gnedin, O. Y., \& Ostriker, J. P. 1999, ApJ, 513, 626

Grillmair, C. J., Freeman, K. C., Irwin, M., \& Quinn, P. J. 1995, AJ, 109, 2553

Grillmair, C. J., \& Johnson, R. 2006, ApJ, 639, L17

Harris, W. E. 1996, AJ, 112, 1487

Illingworth, G., \& Illingworth, W. 1976, ApJS, 30, 227
Johnston, K. V., Sigurdsson, S., \& Hernquist, L. 1999, MNRAS, 302,771

King, I. R. 1966, AJ, 71, 64

Kiss, L. L., Székely, P., Bedding, T. R., Bakos, G. Á., \& Lewis, G. F. 2007, ApJ, 659, L129

Koch, A., Grebel, E. K., Odenkirchen, M., Martínez-Delgado, D., \& Caldwell, J. A. R. 2004, AJ, 128, 2274

Lauchner, A., Powell, W. L. J., \& Wilhelm, R. 2006, ApJ, 651, L33

Lee, K. H., Lee, H. M., Fahlman, G. G., \& Lee, M. G. 2003, AJ, 126,815

Lee, K. H., Lee, H. M., Fahlman, G. G., \& Sung, H. 2004, AJ, 128,2838

Leon, S., Meylan, G., \& Combes, F. 2000, A\&A, 359, 907

McLaughlin, D. E., \& van der Marel, R. P. 2005, ApJS, 161, 304

Milone, A. P., et al. 2008, ApJ, 673, 241

Milone, A. P., Stetson, P. B., Piotto, G., Bedin, L. R., Anderson,

J., Cassisi, S., \& Salaris, M. 2009, arXiv:0906.1779

Newberg, H. J., Yanny, B., \& Willett, B. A. 2009, ApJ, 700, L61

Odenkirchen, M., et al. 2001, ApJ, 548, L165

Odenkirchen, M., et al. 2003, AJ, 126, 2385 
Peñarrubia, J., Navarro, J. F., \& McConnachie, A. W. 2008, ApJ, 673,226

Peterson, C. J., \& King, I. R. 1975, AJ, 80, 427

Piatek, S., 2009, private communication

Ripepi, V., et al. 2007, ApJ, 667, L61

Rockosi, C. M., et al. 2002, AJ, 124, 349

Saha, A., Olszewski, E.W., et al. in preparation

Schechter, P. L., Mateo, M., \& Saha, A. 1993, PASP, 105, 1342

Scholz, R.-D., Irwin, M., Odenkirchen, M., \& Meusinger, H. 1998, A\&A, 333, 531

Sohn, Y.-J., et al. 2003, AJ, 126, 803

Spitzer, L. J. 1940, MNRAS, 100, 396
Testa, V., Zaggia, S. R., Andreon, S., Longo, G., Scaramella, R., Djorgovski, S. G., \& de Carvalho, R. 2000, A\&A, 356, 127

Ventura, P., Caloi, V., D'Antona, F., Ferguson, J., Milone, A., \& Piotto, G. 2009, arXiv:0907.1765

Walker, A. R. 1992, PASP, 104, 1063

Wilson, C. P. 1975 , AJ, 80, 175

Yong, D., Grundahl, F., D'Antona, F., Karakas, A. I., Lattanzio, J. C., \& Norris, J. E. 2009, ApJ, 695, L62

Zoccali, M., Pancino, E., Catelan, M., Hempel, M., Rejkuba, M., \& Carrera, R. 2009, ApJ, 697, L22 